\title{
Virgo Early-Type Dwarfs in ALFALFA
}

\author{
Rebecca A. Koopmann ${ }^{1,2}$ \\ ${ }^{1}$ Department of Physics and Astronomy, Union College, Schenectady, NY 12308, \\ email: koopmanr@union.edu \\ ${ }^{2}$ National Astronomy and Ionosphere Centert, Space Sciences Building, Cornell University, \\ Ithaca NY 14853
}

\begin{abstract}
Early-type dwarf galaxies dominate cluster populations, but their formation and evolutionary histories are poorly understood. The ALFALFA (Arecibo Legacy Fast ALFA) survey has completed observations of the Virgo Cluster in the declination range of 6 - 16 degrees. Less than $2 \%$ of the early-type dwarf population is detected, a significantly lower fraction than reported in previous papers based on more limited samples. In contrast $\sim 30 \%$ of the irregular/BCD dwarf population is detected. The detected early-type galaxies tend to be located in the outer regions of the cluster, with a concentration in the direction of the M Cloud. Many show evidence for ongoing/recent star formation. Galaxies such as these may be undergoing morphological transition due to cluster environmental effects.
\end{abstract}

Keywords. galaxies: dwarf, galaxies: evolution, galaxies: formation, galaxies: clusters: Virgo

\section{Introduction}

Early-type dwarfs are often modeled as forming their stars in a single burst early in the history of the Universe. Yet some early-type dwarfs show signs of relatively recent star formation, as revealed by stellar populations, star formation, and gas content (e.g., Conselice et al. 2003; Lisker et al. 2006). The detection of recent star formation in some systems suggest that at least some early-type dwarfs have formed by transitioning from another galaxy class, e.g., a later-type spiral or dwarf that loses its gas as it enters the hostile environment of a galaxy cluster. Lisker et al. (2007) find that approximately half of the early-type dwarfs in Virgo belong to an unrelaxed population that could be associated with recently infalling galaxies.

Conselice et al. (2003) suggested that up to $15 \%$ of Virgo Cluster dE galaxies have an HI reservoir. However this study was based on observations of a total of only 48 objects and used results from a heterogeneous set of telescopes. The Arecibo Legacy Fast ALFA (ALFALFA) Survey, a sensitive blind survey of the Arecibo sky (Giovanelli et al. 2005 and these proceedings), is providing a complete and unbiased view of HI content and structures in the entire Virgo cluster region.

\section{Observations and Results}

ALFALFA detections in the Virgo Cluster between the declinations of 6 - 16 degrees (Giovanelli et al. 2007; Kent et al. 2007, in prep., Koopmann et al. 2007, in prep.) were compared with the Virgo Cluster Catalog (VCC: Binggeli, Sandage, \& Tammann 1985; Binggeli, Popescu, \& Tammann 1993) to identify galaxies classified as early-type dwarfs. A total of 16 or $1.4 \%$ of the $\mathrm{dE} / \mathrm{dS} 0$ in this declination range were detected by ALFALFA. This compares to a detection rate of $29 \%$ for dwarf irregulars and $27 \%$ for

$\dagger$ The National Astronomy and Ionosphere Center is operated by Cornell University under a cooperative agreement with the National Science Foundation. 

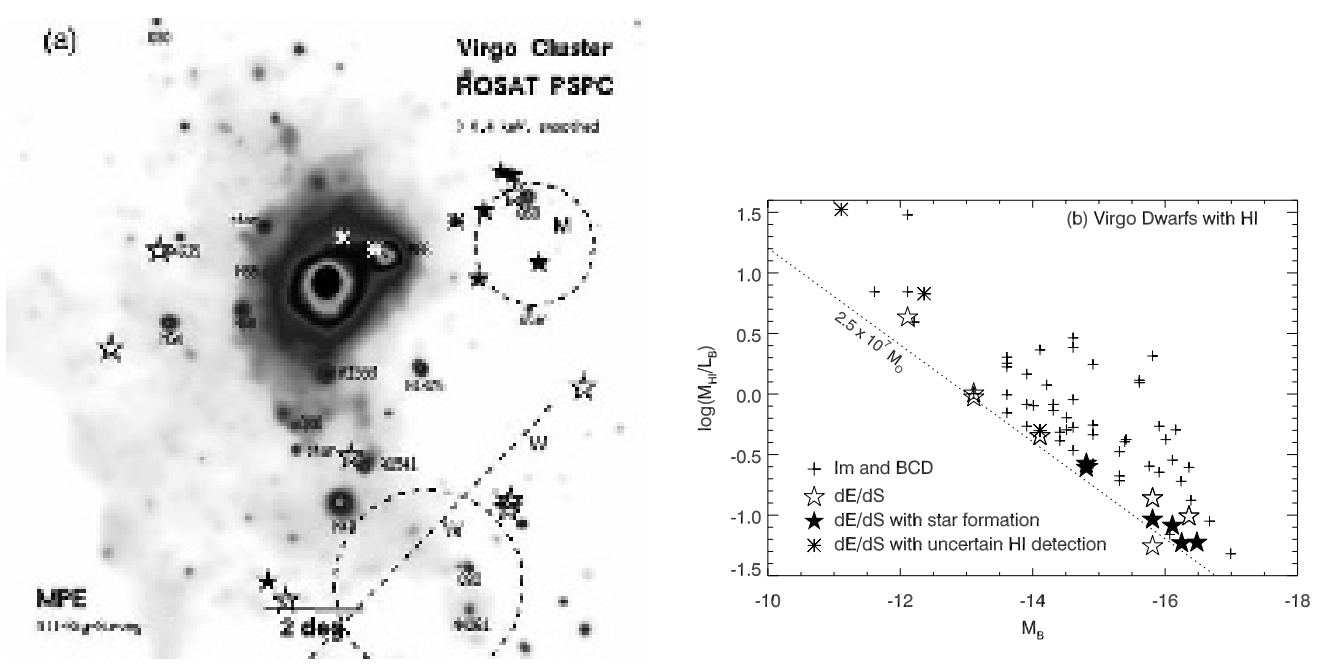

Figure 1. (a) Positions of Virgo early-type dwarfs overlaid on ROSAT greyscale (Boehringer et al. 1994), with $\mathrm{M}, \mathrm{W}$, and $\mathrm{W}^{\prime}$ cloud positions after Binggeli et al. (1993). (b) $M_{H I} / L_{B}$ as a function of $M_{B}$ for Virgo dwarfs with HI detected by ALFALFA. A key to the symbols is given in the legend.

BCDs. $63 \%$ of the early type detections are new HI measurements and $32 \%$ are new redshift measurements. $56 \%$ have peculiar or uncertain classification. Most are located in the cluster outskirts (Figure 1a), with several in the direction of the M Cloud. Six of nine observed at $\mathrm{H} \alpha$ wavelengths show emission due to star formation.

Conselice et al. (2003) reported 7/48 (15\%) Virgo dE/dS0 sample detected in HI. Of the five within our declination range, two, the brightest in their sample, are not detected by ALFALFA and were likely confused in Conselice et al. Three are reproduced by ALFALFA with comparable masses, but two of these, VCC 31 (classified '?' in the VCC) and VCC 2062, are of uncertain type and are not counted here as dE/dS0.

The median HI mass of the detected early-types is $3.2 \times 10^{7} M_{\odot} \quad$ (which is only 1.6 times the ALFALFA detection limit at the Virgo distance of $16.7 \mathrm{Mpc}$ ) and the median $\mathrm{M}_{H I} / \mathrm{L}_{B}$ is 0.14 . They have lower $\mathrm{M}_{H I} / \mathrm{L}_{B}$ than Virgo dwarfs classified as $\mathrm{Im}$ or BCD (Figure 1b). Virgo dwarfs spread to lower $\mathrm{M}_{H I} / \mathrm{L}_{B}$ than more isolated dwarfs. Detailed results are presented in Koopmann et al. (2007, in prep.).

\section{Acknowledgements}

The author is grateful for partial support from NAIC, travel support from the Mellon Foundation, and for the hospitality of the Cornell University Astronomy Department during a sabbatic visit.

\section{References}

Binggeli, B., Sandage, A., \& Tammann, G. A. 1985, AJ 90, 1681

Binggeli, B., Popescu, C. C., \& Tammann, G. A. 1993, A\& AS, 98, 275

Boehringer, H. U. G. et al. 1994, Nature, 368, 828

Conselice, C. J., O’Neil, K., Gallagher, J. S., III, \& Wyse, R. F. G. 2003, ApJ, 591, 167

Giovanelli, R et al. 2007, AJ, 133, 2569

Giovanelli et al. 2005a, AJ, 130, 2598

Lisker, T., Glatt, K., Westera, P., \& Grebel, E. K. 2006, AJ 132, 2432

Lisker, T., Grebel, E. K., Binggeli, B., \& Glatt, K. 2007, ApJ, 660, 1186 